[9] D. Chen and T. Saito, "A new method to reduce the complexity of joint detection algorithm," in Proc. GLOBECOM, San Francisco, CA, Dec. 2003, pp. 2335-2339.

[10] Tdoc SMG2 UMTS L1, ETSI Std. 362/98, Sep. 1998.

[11] Physical Channels and Mapping of Transports Channels Onto Physical Channels (TDD), Dec. 2002. 3rd Generation Partnership Project (3GPP) Technical Specification Group Radio Access Network 3GPP TS 25.221, Rev. 4.7.

[12] Spreading and Modulation (TDD), Dec. 2002. 3rd Generation Partnership Project (3GPP) Technical Specification Group Radio Access Network 3GPP TS 25.223, Rev. 4.5.

[13] J. Shynk, "Frequency-domain and multirate adaptive filtering," IEEE Signal Process. Mag., vol. 9, no. 1, pp. 14-37, Jan. 1992.

[14] D. Falconer and S. L. Ariyavisitakul, "Broadband wireless using single carrier and frequency domain equalization," in Proc. 5th Int. Symp. Wireless Pers. Multimedia Commun., Oct. 2002, vol. 1, pp. 27-36.

[15] L. Goncalves and A. Gameiro, "Frequency domain equalizer for multirate UMTS-TDD systems," in Proc. ICC, May 2003, vol. 5, pp. 3236-3240.

[16] L. Martoyo, T. Weiss, T. F. Capar, and F. K. Jondral, "Low complexity CDMA downlink receiver based on frequency domain equalization," in Proc. VTC-Fall, Oct. 2003, vol. 2, pp. 987-991.

\section{Iterative Near-Maximum-Likelihood Detection in Rank-Deficient Downlink SDMA Systems}

\author{
Chun-Yi Wei, Jos Akhtman, Soon Xin Ng, and Lajos Hanzo
}

\begin{abstract}
In this paper, a precoded and iteratively detected downlink multiuser system is proposed, which is capable of operating in rankdeficient scenarios, when the number of transmitters exceeds the number of receivers. The literature of uplink space division multiple access (SDMA) systems is rich, but at the time of writing there is a paucity of information on the employment of SDMA techniques in the downlink. Hence, we propose a novel precoded downlink SDMA (DL-SDMA) multiuser communication system, which invokes a low-complexity nearmaximum-likelihood sphere decoder and is particularly suitable for the aforementioned rank-deficient scenario. Powerful iterative decoding is carried out by exchanging extrinsic information between the precoder's decoder and the outer channel decoder. Furthermore, we demonstrate with the aid of extrinsic information transfer charts that our proposed precoded DL-SDMA system has a better convergence behavior than its nonprecoded DL-SDMA counterpart. Quantitatively, the proposed system having a normalized system load of $L_{s}=1.333$, i.e., 1.333 times higher effective throughput facilitated by having 1.333 times more DL-SDMA transmitters than receivers, exhibits a "turbo cliff" at an $E_{b} / N_{0}$ of $5 \mathrm{~dB}$ and hence results in an infinitesimally low bit error rate (BER). By contrast, at $E_{b} / N_{0}=5 \mathrm{~dB}$, the equivalent system dispensing with precoding exhibits a BER in excess of $10 \%$.
\end{abstract}

Index Terms-Iterative decoding, maximum likelihood detection, space division multiple access (SDMA) downlink, sphere decoding.

\section{INTRODUCTION}

Multiple-input-multiple-output (MIMO) systems employing multiple antennas at both the transmitter and receiver exhibit a substantially higher spectral efficiency than conventional single-antenna systems. The flexible configuration of a MIMO system's antennas allows us to satisfy a number of potentially contradictory design objectives in terms

Manuscript received August 14, 2006; revised December 21, 2006, March 7, 2007, and March 9, 2007. This work was supported in part by the European Union under the auspices of the Phoenix and Newcom projects and in part by the Engineering and Physical Sciences Research Council, U.K. The review of this paper was coordinated by Dr. A. Ghrayeb.

The authors are with the School of Electronics and Computer Science, University of Southampton, Southampton SO17 1BJ, U.K. (e-mail: lh@ecs.soton.ac.uk).

Digital Object Identifier 10.1109/TVT.2007.901879 of the achievable multiplexing and diversity gain, hence this topic has recently attracted substantial research attention [1], [2]. Space division multiple access (SDMA) constitutes an attractive MIMO subclass, which is capable of achieving a high user capacity by supporting a multiplicity of subscribers within the same frequency bandwidth [3], [4]. ${ }^{1}$

The efficient design of the downlink transmitter is of paramount importance for the sake of achieving a high throughput. The effects of a multiuser interference (MUI) may be mitigated by employing a spatiotemporal preproccessing at the transmitter. Consequently, the downlink receiver's complexity may be reduced with the advent of transmit preprocessing at the base station (BS), a technique that is also often referred to as the multiuser transmission (MUT) [7].

Furthermore, in the context of MUT, time division duplexing is often invoked for separating the uplink and downlink traffic. This is because when the channel's impulse response is assumed to be both quasi-stationary and at the same time similar in the uplink and downlink, we may assume that the channel transfer function that is estimated in the uplink may be used for spatiotemporal preprocessing in the downlink. A witty approach to spatiotemporal preproccessing was proposed by Vandenameele et al. [4], where the MUT transformation matrix was specifically designed so that its product with the channel matrix yielded an identity matrix. This MUT transformation matrix may be regarded as a perfect preequalizer, which effectively results in an MUI-free channel. Furthermore, Choi and Murch [8] proposed an attractive MUT design ${ }^{2}$ that allows for a specific user to receive his/her dedicated signal, which is entirely free from an MUI that is inflicted by other users, provided that a perfect knowledge of each of the MIMO links is available at the transmitter. A somewhat similar preprocessing method, which is referred to as the block diagonalization algorithm, was discussed in [10], which relied on employing the singular value decomposition (SVD). More specifically, the spatiotemporal preprocessing technique in [8] decomposes a MIMO channel into a set of parallel single-user MIMO channels, which facilitates the employment of well-known MIMO-processing techniques [3], [4].

However, the performance of the family of classic linear detectors, such as the minimum mean square error (MMSE) detector [3], was shown to be unsatisfactory in high-throughput rank-deficient scenarios [11]. As a solution, nonlinear (NL) detectors may be used [3]. However, the typical high complexity of NL detectors [3] is often prohibitive in practical systems. Thus, reduced search algorithms (RSA) may be employed for mitigating the complexity of the NL detector. A novel optimized hierarchy RSA (OHRSA)-aided maximum-likelihood (ML) detection method was advocated in [11], which may be regarded as an advanced extension of the complex-valued sphere decoding (CSD) techniques that were depicted in [12]. As opposed to the CSD, the OHRSA exhibits a relatively low complexity even in highly rankdeficient scenarios, and thus, its employment is meritorious.

Since there are no in-depth near-ML downlink SDMA (DL-SDMA) rank-deficient system studies in the open literature, the novel contribution of this paper is that the low-complexity near-ML uplink OHRSA detector in [11] is invoked for a rank-deficient DL-SDMA multiuser system, which is capable of receiving from more transmitters than the number of receivers. Furthermore, the convergence of the iterative detector is improved with the aid of precoding by exchanging extrinsic information between the constituent decoders and investigated using extrinsic information transfer (EXIT) charts.

\footnotetext{
${ }^{1}$ Other MIMO systems such as beamformers [5] and space-time codes [6] are not considered in this contribution.

${ }^{2}$ Choi and Murch [8] utilized an MUT design for eliminating the MUI, which they also refer to as a transmit precoder. By contrast, in this paper, the precoder is a unity-rate convolutional encoder that uses a single shift register stage [9].
} 


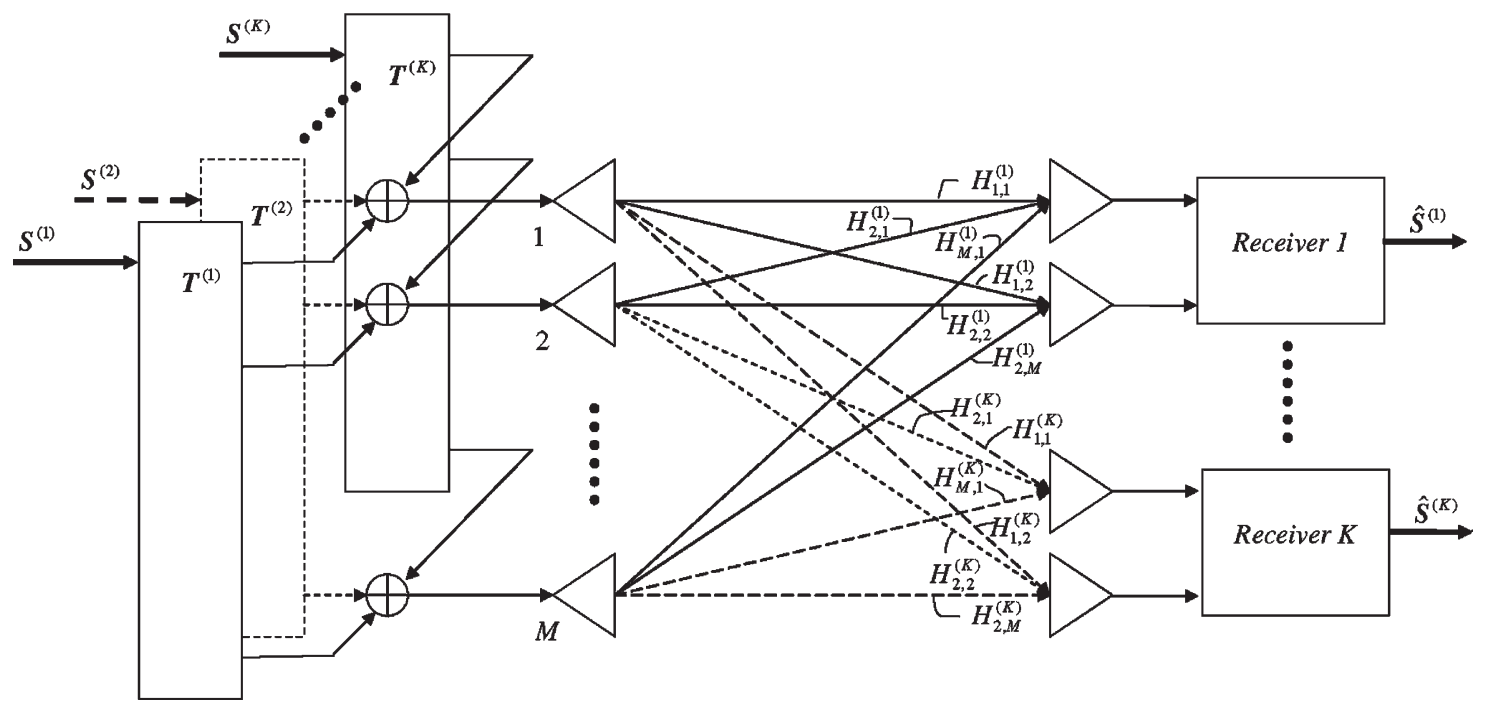

Base Station

Fig. 1. MUT in the DL-SDMA system.

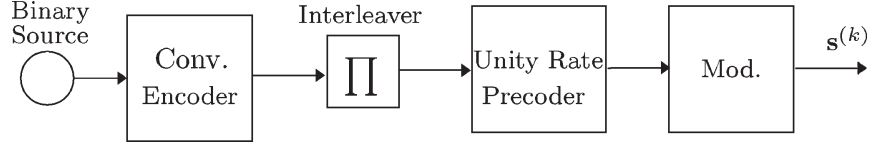

Fig. 2. Generating the precoded data symbols for the $k$ th user.

The rest of this paper is structured as follows: In Section II, we outline the system model used, whereas in Section III, the normalized system throughput is discussed. The OHRSA detector is summarized in Section IV, followed by the description of the iterative decoding algorithm in Section V. Our EXIT chart analysis is provided in Section VI, leading to the performance results provided in Section VII. Finally, we conclude our discourse in Section VIII.

\section{SYSTEM MODEL}

The DL-SDMA system considered in this paper is depicted in Fig. 1. More specifically, our system comprises a BS employing $M$ transmit antennas and $K$ mobile stations (MSs), where each of the MSs employs $N_{k}$ receive antennas. In this paper, we consider a flatfading MIMO channel. Consequently, each link between the $i$ th BS transmit antenna and the $j$ th MS receive antenna of the $k$ th user may be characterized by a complex-valued scalar channel coefficient $H_{i j}^{(k)}$, which we assume to be an independent identically distributed Gaussian random variable having a variance of unity and a mean of zero. Moreover, the MIMO channel corresponding to the $k$ th user may be described as an $\left(N_{k} \times M\right)$-dimensional complex-valued timedomain channel matrix $\mathbf{H}^{(k)}$, which may be defined as follows:

$$
\mathbf{H}^{(k)}=\left(\begin{array}{cccc}
H_{1,1}^{(k)} & H_{1,2}^{(k)} & \cdots & H_{1, M}^{(k)} \\
H_{2,1}^{(k)} & H_{2,2}^{(k)} & \cdots & H_{2, M}^{(k)} \\
\vdots & \vdots & \cdots & \vdots \\
H_{N_{k}, 1}^{(k)} & H_{N_{k}, 2}^{(k)} & \cdots & H_{N_{k}, M}^{(k)}
\end{array}\right) .
$$

As illustrated in Fig. 2, the data bits are encoded by both the channel encoder and the unity-rate precoder before modulation. More explicitly, the unity-rate precoder is a convolutional encoder that uses a single shift register stage [9]. Let $\mathbf{s}^{(k)} \in \mathbb{C}^{L_{k} \times 1}$ be a complex-valued column vector, which denotes the precoded data symbol vector to be transmitted to the $k$ th MS, while $L_{k}$ represents the number of independent data symbols contained in $\mathbf{s}^{(k)}$. Additionally, we define the so-called space-time preprocessor matrix $\mathbf{T}^{(k)} \in \mathbb{C}^{M \times L_{k}}$, which was designed for the sake of eliminating the MUI [8]. As suggested by Choi and Murch [8], we may formulate a solution of the MUT design problem as $\mathbf{T}^{(k)}=\mathbf{V}^{(k)} \mathbf{A}^{(k)}$, where $\mathbf{A}^{(k)}$ is a nonzero $\left(n_{k} \times L_{k}\right)$-dimensional matrix and $\mathbf{V}^{(k)}$ can be calculated using the SVD [13] of $\tilde{\mathbf{H}}^{(k)}$ that is expressed as

$$
\tilde{\mathbf{H}}^{(k)}=\left(\begin{array}{ll}
\tilde{\mathbf{U}}^{(k)} & \mathbf{U}^{(k)}
\end{array}\right) \cdot\left(\begin{array}{cc}
\Sigma & \mathbf{0} \\
\mathbf{0} & \mathbf{0}
\end{array}\right) \cdot\left(\begin{array}{l}
\tilde{\mathbf{V}}^{(k)^{H}} \\
\mathbf{V}^{(k)^{H}}
\end{array}\right)
$$

while

$$
\tilde{\mathbf{H}}^{(k)}=\left(\begin{array}{llllll}
\mathbf{H}^{(1)} & \ldots & \mathbf{H}^{(k-1)} & \mathbf{H}^{(k+1)} & \ldots & \mathbf{H}^{(K)}
\end{array}\right)^{T} .
$$

Furthermore, let $\mathbf{r}^{(k)}$ and $\mathbf{n}^{(k)}$ be the received signal vector and noise vector associated with the $k$ th MS, respectively. As it was demonstrated in [8], once the MUI was eliminated by the MUT, the received signal vector associated with the $k$ th MS can be expressed in the following form:

$$
\mathbf{r}^{(k)}=\mathbf{H}^{(k)} \mathbf{T}^{(k)} \mathbf{s}^{(k)}+\mathbf{n}^{(k)}
$$

where the $\left(N_{k} \times L_{k}\right)$-dimensional matrix $\mathbf{H}^{(k)} \mathbf{T}^{(k)}$ characterizes the effective channel corresponding to the $k$ th MS. Note that according to (2) the nonzero matrices $\mathbf{T}^{(k)}$ exist only in the scenario when $\tilde{\mathbf{H}}^{(k)}$ has more columns than rows. Consequently, we have to satisfy the following condition:

$$
M>\max \left\{\sum_{i=1, i \neq k}^{K} N_{i}, \quad k=1,2, \ldots, K\right\} .
$$

Moreover, the rank $n_{k}$ of the null space basis $\mathbf{V}^{(k)}$ may be expressed as

$$
n_{k}=M-\sum_{i=1, i \neq k}^{K} N_{i}
$$


It can be observed that the particular value of $n_{k}$ will directly affect the spatial multiplexing gain achievable by the system. The subject of the achievable multiplexing gain as well as the transmit diversity gain will be further explored in Section III.

\section{NORMALIZED SySTEM LOAD $L_{s}$}

The DL-SDMA system considered in this paper provides a flexible system design framework that is capable of supporting various spatial multiplexing and diversity requirements. More specifically, an equivalent single-user SDMA system may potentially provide a spatial multiplexing gain of $\min \left\{n_{k}, N_{k}\right\}$ [1]. Furthermore, in the scenario of having a $K$-user SDMA system, where the corresponding MIMO channel may be decomposed into $K$ number of parallel single-user MIMO channels, the system may potentially achieve a spatial multiplexing gain, which linearly increases with the value of $\min \left\{K n_{k}, K N_{k}\right\}$.

As previously stated, in this paper, we considered a particular scenario of having $L_{k}=n_{k}$, where the corresponding dimension of the effective transmit antenna array that is encountered by the $k$ th MS is $L_{k}$. Consequently, the achievable spatial multiplexing gain will linearly increase with the value of $\min \left\{K L_{k}, K N_{k}\right\}$. Furthermore, the achievable transmit diversity increases with the number of physical transmit antennas $M$. It can be observed that the MUT's preprocessor matrix $\mathbf{T}^{(k)}$ transforms the signal vector $\mathbf{s}^{(k)}$ into the column space of $\mathbf{T}^{(k)}$. Hence, each element of $\mathbf{s}^{(k)}$ contributes to each of the signals that are transmitted from the corresponding physical transmit antennas. For instance, let $x_{i}$ denote the symbol transmitted from the $i$ th transmit antenna. Then, $x_{i}, i=1, \ldots, M$, comprises the contributions from each element of the transmit symbol vector $\mathbf{s}^{(k)}$, which results in the transmit diversity gain achievable by the system.

In the scenario of having an $(M \times N)$-dimensional MIMO channel, where $M$ and $N$ refer to the number of transmit antennas and the total number of antennas employed by all user terminals, respectively, different system configurations of $M$ and $N$ provide different spatial multiplexing and transmit diversity gains. To categorize the potential system design options relevant to the current discussion, let us introduce the measure of the normalized system load that is expressed as

$$
L_{s}=\frac{M}{N} .
$$

Consequently, we may distinguish three different scenarios as follows:

1) lightly loaded scenario, for $L_{s}<1$;

2) fully loaded scenario, for $L_{s}=1$;

3) rank-deficient or "over-loaded" scenario, for $L_{s}>1$.

In the lightly loaded case, the number of antennas in the receiver exceeds that in the transmitter. Hence, the extra receiver antennas may potentially provide the system with the corresponding receive diversity. On the other hand, in the fully loaded case, the receiver antennas provide a degree of freedom, which is just sufficient for a linear detector to separate the $M$ independent users' signals. Finally, in the rank-deficient scenario, the number of receiver antennas is insufficient for providing an adequate degree of freedom required by a linear detector. Thus, NL detection techniques have to be employed. The normalized system load $L_{s}$ of (7) may be utilized to characterize both uplink SDMA and DL-SDMA systems.

\section{OHRSA-AIDED ML DETECTION}

In this section, we briefly summarize the principles of the OHRSA [11], which we adopted for our DL-SDMA system to reduce the computational complexity imposed by the ML detector employed by each of the MSs. For the sake of convenience, in our forthcoming discussions, we will focus on a single MS and omit the user index $k$. The optimum ML solution is given by [3]

$$
\hat{\mathbf{s}}=\arg \min _{\breve{\mathbf{s}} \in \mathcal{M}^{L}}\|\mathbf{U}(\breve{\mathbf{s}}-\hat{\mathbf{x}})\|^{2}
$$

where $\mathcal{M}$ denotes the constellation size of the modulation scheme employed, and $\mathcal{M}^{L}$ is the total set of legitimate values hosted by the transmitted symbol vector $\breve{\mathbf{s}}$. Moreover, $\mathbf{U}$ is an upper triangular matrix having real-valued elements on its main diagonal and satisfying

$$
\mathbf{U}^{H} \mathbf{U}=\left(\mathbf{H}_{e}^{H} \mathbf{H}_{e}+\sigma_{n}^{2} \mathbf{I}\right)
$$

while

$$
\hat{\mathbf{x}}=\left(\mathbf{H}_{e}^{H} \mathbf{H}_{e}+\sigma_{n}^{2} \mathbf{I}\right)^{-1} \mathbf{H}_{e}^{H} \mathbf{r}
$$

and the effective channel matrix is given by $\mathbf{H}_{e}=\mathbf{H} \cdot \mathbf{T}$, where again we omit the user index $k$ for the sake of brevity. Consequently, let us define the following objective function [11]:

$$
\begin{aligned}
\mathcal{J}(\breve{\mathbf{s}}) & =\|\mathbf{U}(\breve{\mathbf{s}}-\hat{\mathbf{x}})\|^{2}=(\breve{\mathbf{s}}-\hat{\mathbf{x}}) \mathbf{U}^{H} \mathbf{U}(\breve{\mathbf{s}}-\hat{\mathbf{x}}) \\
& =\sum_{i=1}^{L}\left|\sum_{j=i}^{L} u_{i j}\left(\breve{s}_{j}-\hat{x}_{j}\right)\right|^{2}=\sum_{i=1}^{L} \phi_{i}\left(\breve{\mathbf{s}}_{i}\right) \\
\mathcal{J}_{i}\left(\breve{\mathbf{s}}_{i}\right) & =\mathcal{J}_{i+1}\left(\breve{\mathbf{s}}_{i+1}\right)+\phi_{i}\left(\breve{\mathbf{s}}_{i}\right), \quad i=1, \ldots, L
\end{aligned}
$$

where we have $\breve{\mathbf{s}}_{i}=\left(\breve{s}_{i}, \ldots, \breve{s}_{L}\right)$ and

$$
\mathcal{J}(\breve{\mathbf{s}})=\mathcal{J}_{1}\left(\breve{\mathbf{s}}_{1}\right)>\mathcal{J}_{2}\left(\breve{\mathbf{s}}_{2}\right)>\cdots>\mathcal{J}_{L}\left(\breve{\mathbf{s}}_{L}\right)>0 .
$$

Equations (12) and (13) enable us to employ a highly efficient reduced-complexity search algorithm, which decreases the number of objective function evaluations associated with solving the minimization problem of (8) to a small fraction of the entire set $\mathcal{M}^{L}$.

The derivation of an expression for the low-complexity evaluation of the soft-bit information that is associated with the bit estimates of the detector's output as characterized by (8) is given in [11]. Specifically, it is demonstrated in [11] that the soft-bit value associated with the $m$ th bit of the $i$ th quadrature-amplitude modulation (QAM) symbol of the data symbol vector assigned to the $k$ th user may be closely approximated as

$$
L_{i m}^{(k)} \approx \frac{1}{\sigma_{n}^{2}}\left[\mathcal{J}\left(\breve{\mathbf{s}}_{i m ; \min }^{0}\right)-\mathcal{J}\left(\breve{\mathbf{s}}_{i m ; \min }^{1}\right)\right]
$$

where both $\check{\mathbf{s}}_{i m \text {;min }}^{b}$ and the corresponding cost function value $J\left(\check{\mathbf{s}}_{i m ; \text { min }}^{b}\right)$ may be obtained by applying the extended OHRSA-aided search that was derived in [11] and briefly summarized in the previous discussion.

\section{ITERATIVE DECODING}

Iterative decoding is carried out by exchanging extrinsic information between the unity-rate precoder and the channel decoder. Fig. 3 illustrates the iterative receiver structure, where $L$ represents the $\log$ likelihood ratios (LLRs). The superscript Det indicates the detector, $\mathrm{P}$ denotes the precoder, and Dec represents the channel decoder. The subscripts apr, ex, and apt indicate a priori, extrinsic, and 


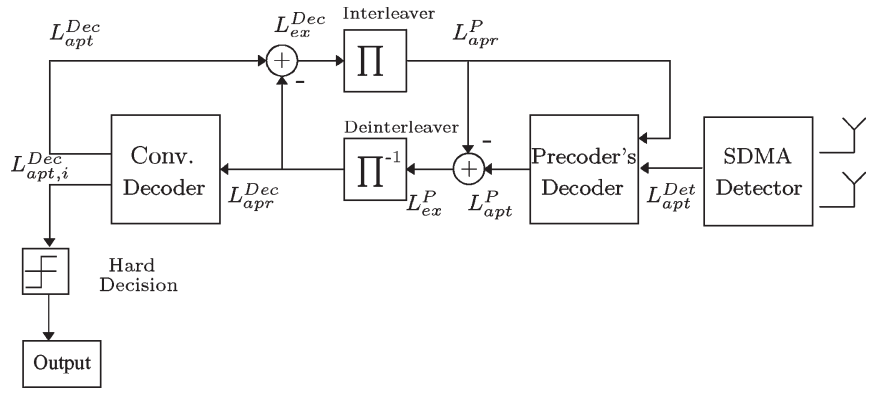

Fig. 3. Iteratively decoded receiver design.

TABLE I

SYSTEM CONFIGURATIONS INVESTIGATED IN Fig. 5

\begin{tabular}{|c|c|c|c|c|c|}
\hline$M$ & 6 & 7 & 8 & 9 & 10 \\
\hline$L$ & 2 & 3 & 4 & 5 & 6 \\
\hline$L_{s}$ & 1 & 1.167 & 1.333 & 1.5 & 1.667 \\
\hline
\end{tabular}

a posteriori LLRs, respectively. First, the unity-rate precoder's decoder in Fig. 3 processes the soft-bit output $L_{\text {apt }}^{\text {Det }}$ of the detector that is generated in the previous stage, and the a priori LLR values $L_{\mathrm{apr}}^{\mathrm{P}}$, which are appropriately arranged by the interleaver $\Pi$, are produced from the extrinsic information $L_{\mathrm{ex}}^{\mathrm{Dec}}$ of the channel decoder. Then, the extrinsic information $L_{\mathrm{ex}}^{\mathrm{P}}$ to be used by the unity-rate precoder's decoder is obtained from $L_{\text {apt }}^{\mathrm{P}}$ by subtracting the a posteriori LLR values $L_{\text {apr }}^{\mathrm{P}}$, as shown in Fig. 3. Then, the channel decoder processes $L_{\mathrm{apr}}^{\mathrm{Dec}}$, which was generated by the deinterleaver $\Pi^{-1}$ from $L_{\mathrm{ex}}^{\mathrm{P}}$, and outputs the a posteriori LLRs $L_{\text {apt }}^{\text {Dec }}$ to be used as a feedback for the next decoding iteration. When the iterations are curtailed, the channel decoder outputs $L_{\mathrm{apt}, i}^{\mathrm{Dec}}$, which represents the hard-decision-based data bits. In Section VI, we will use EXIT charts [14] in our detailed investigations of the iterative receiver.

\section{EXIT CHART ANALYSIS}

The system used the low-complexity OHRSA detector in Section IV, which employed a 4-QAM and a half-rate recursive systematic convolutional (RSC) coding having a memory of 3 as well as an octal generator polynomial of $G=[57]$. The system configuration and the corresponding normalized system loads are detailed in Table I.

According to [14], if the extrinsic transfer curves intersect at the $\left(I_{\mathrm{apr}}^{\mathrm{det}}, I_{\mathrm{ex}}^{\mathrm{det}}\right)=(1.0,1.0)$ point in Fig. 4 and an open EXIT tunnel exists at a certain SNR, then the system will exhibit an infinitesimally low bit error rate (BER). In Fig. 4, the system operates at an SNR of $11 \mathrm{~dB}$. It can be observed that the nonprecoded DL-SDMA system does not exhibit an open EXIT tunnel, despite emerging from a higher $I_{\mathrm{apr}}^{\mathrm{dec}}$ point on the vertical axis than its precoded counterpart. More explicitly, its EXIT curve intersects with that of the RSC $(5,7)$ code at a point lower than $\left(I_{\mathrm{apr}}^{\mathrm{det}}, I_{\mathrm{ex}}^{\mathrm{det}}\right)=(1.0,1.0)$. Therefore, the nonprecoded DL-SDMA system operating at an SNR of $11 \mathrm{~dB}$ is expected to exhibit a rather high BER. On the other hand, at the same SNR of $11 \mathrm{~dB}$, the precoded system exhibits an open EXIT tunnel, despite emerging from a lower $I_{\text {apr }}^{\text {dec }}$ point, since it intersects the EXIT curve of the $\operatorname{RSC}(5,7)$ code at the $\left(I_{\mathrm{apr}}^{\mathrm{det}}, I_{\mathrm{ex}}^{\text {det }}\right)=(1.0,1.0)$ point. Hence, the precoded DL-SDMA system has a better iterative decoding convergence than the nonprecoded system, consequently exhibiting a better BER performance.

Furthermore, according to the actual decoding trajectory of the proposed precoded and OHRSA-decoded DL-SDMA system illustrated in Fig. 4, $I=8$ iterations are required for maintaining an open EXIT

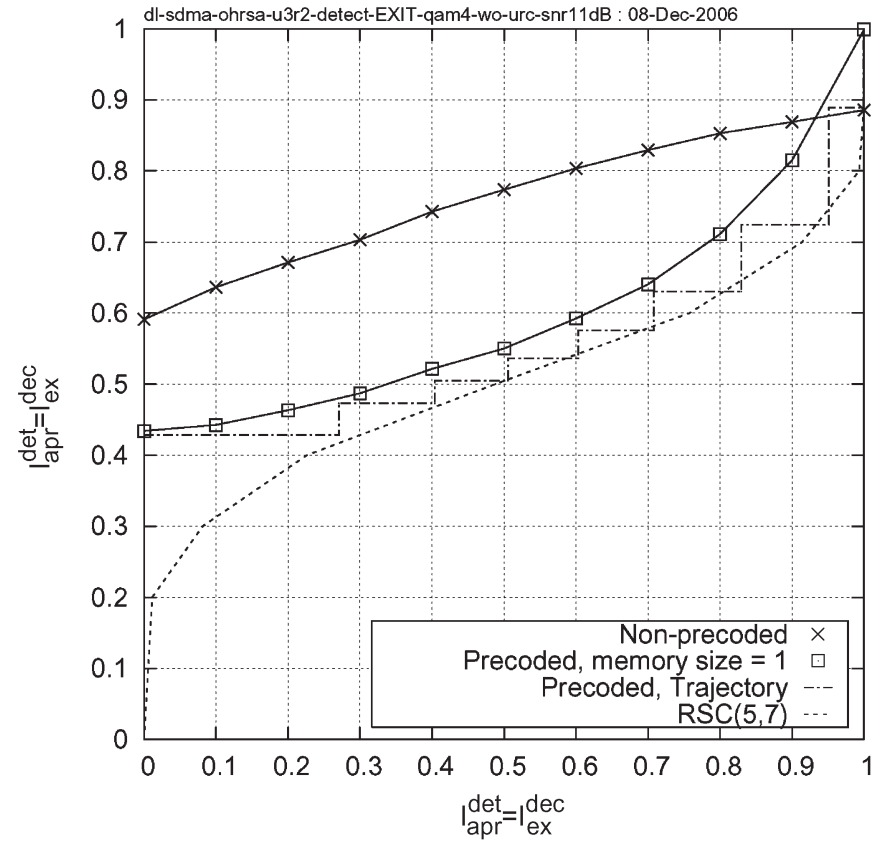

Fig. 4. EXIT chart comparison of the nonprecoded and precoded DL-SDMA systems having a normalized system load of $L_{s}=1.333$ at $\mathrm{SNR}=11 \mathrm{~dB}$. This system supports $K=3$ users, where each user employs $N_{k}=2$ receive antennas.

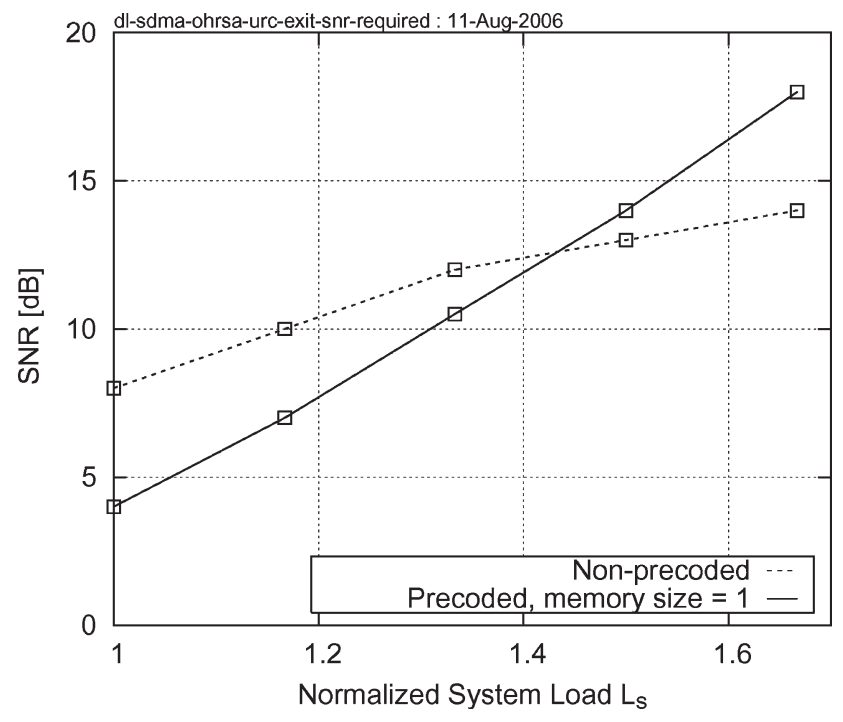

Fig. 5. SNR required by both the nonprecoded and precoded DL-SDMA systems to exhibit an open EXIT tunnel at different system loads. The five points recorded correspond to the system configurations of $\left(M, N_{k}\right)=$ $[(6,2)(7,3)(8,4)(9,5)(10,6)]$ in Table I.

tunnel, when the precoded DL-SDMA OHRSA detector employs an interleaver length of $10^{5}$ bits.

Fig. 5 shows the minimum SNR required by both the nonprecoded and the precoded DL-SDMA systems to exhibit open EXIT tunnels at different normalized system loads. When the systems operate at those SNRs, we expect to see the emergence of turbo cliffs in the corresponding BER curves. As observed in Fig. 5, a lower SNR is required for the precoded DL-SDMA system to exhibit a turbo cliff than by the nonprecoded DL-SDMA system, when the normalized system load is lower than $L_{s}=1.333$. Beyond the corresponding SNRs, the systems are capable of operating at an infinitesimally 


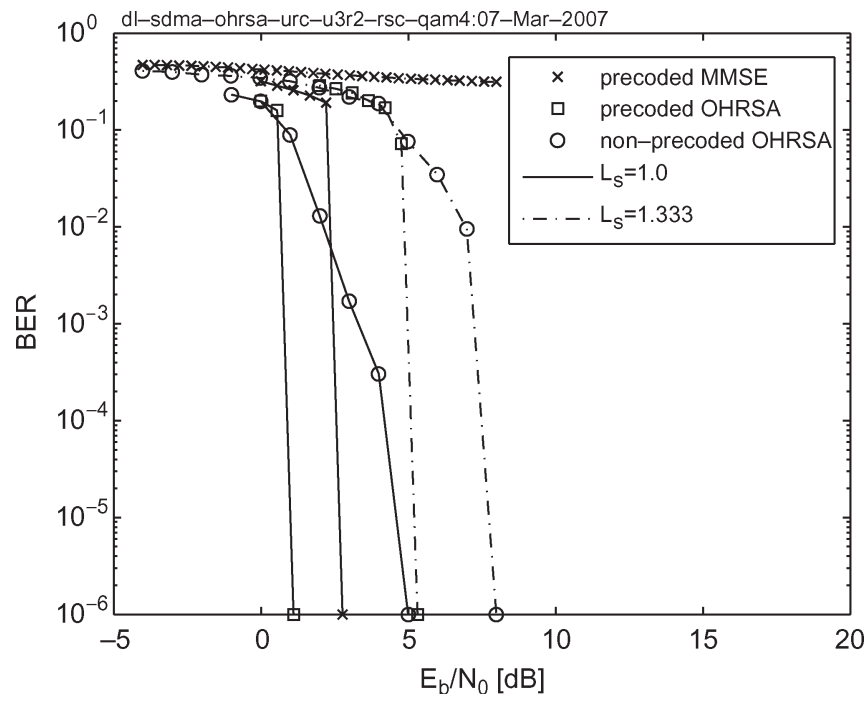

Fig. 6. BER performance of the precoded DL-SDMA scheme having a normalized system load of $L_{s}=1.0$ and 1.333. Our system supports $K=3$ users, where each user employs $N_{k}=2$ receive antennas. The length of the interleaver used is $10^{5}$, and $I=10$ iterations are employed by the iterative decoder. The channel model was a flat-fading MIMO channel.

low BER. By contrast, when the normalized system load is increased beyond 1.333, the precoded system requires a high SNR for maintaining an open EXIT tunnel associated with an infinitesimally low BER.

\section{BER PERFORMANCE}

Finally, in Fig. 6, we characterize the achievable BER performance of the precoded DL-SDMA system employing the OHRSA detector. Again, we employed the 4-QAM that is protected by the half-rate $\operatorname{RSC}(5,7)$ code having a memory of 3 and using a $10^{5}$-bit interleaver. The channel model was a flat-fading MIMO channel. All system configurations considered supported $K=3$ users. Finally, for the sake of convenience, we assume $L_{1}=L_{2}=\ldots=L_{k}=L$.

Fig. 6 shows our BER performance results that corresponds to two different system load scenarios, namely $L_{s}=1.0$ and 1.333. The system configuration and the corresponding normalized system loads are detailed in Table I. For each system load scenario, we characterize the BER performance of the precoded DL-SDMA system invoking the OHRSA detector along with that of the precoded DL-SDMA system employing the MMSE detector. The nonprecoded DL-SDMA system employing the OHRSA detector is also characterized in Fig. 6 as a benchmark. In the fully loaded scenario of $L_{s}=1.0$, all three detectors exhibit an adequate performance. However, the precoded DL-SDMA system using both the MMSE and the OHRSA detectors outperforms the nonprecoded OHRSA-aided system. The precoded DL-SDMA system using the MMSE detector has a 2-dB $E_{b} / N_{0}$ gain over the nonprecoded DL-SDMA OHRSAaided system at a target BER of $10^{-5}$. At the same time, the $E_{b} / N_{0}$ required by the precoded DL-SDMA OHRSA-aided system for maintaining a target BER of $10^{-5}$ is $2 \mathrm{~dB}$ lower than that of the MMSE detector.

Furthermore, when the normalized system load is increased to $L_{s}=1.333$, which corresponds to a highly rank-deficient scenario, the MMSE detector fails to attain a satisfactory BER performance. On the other hand, both the nonprecoded and precoded DL-SDMA OHRSA-aided systems perform well. However, the $E_{b} / N_{0}$ needed by the precoded system for achieving a target BER of $10^{-5}$ is $2 \mathrm{~dB}$ lower than that needed by the OHRSA-aided nonprecoded system for attaining the same target BER. At an $E_{b} / N_{0}$ of $4 \mathrm{~dB}$, the precoded OHRSA-aided DL-SDMA system exhibits a turbo cliff and results in an infinitesimally low BER, while the nonprecoded system supporting the same system load exhibits a BER in excess of $10 \%$.

\section{CONCLUSION}

Although the uplink performance of SDMA systems is well documented, there is a paucity of DL-SDMA studies, and no in-depth studies can be found in the open literature for high-throughput rankdeficient systems. In this scenario, low-complexity linear detectors, such as the MMSE detector, exhibit a high residual error floor, while most NL detectors exhibit an excessive complexity. Hence, the near-ML OHRSA detector was adopted for employment in the downlink, and it was amalgamated with an iteratively detected unity-rate precoder. This amalgamated and iteratively detected rankdeficient system was capable of achieving an infinitesimally low BER at a normalized system load of $L_{s}=1.333$ and $E_{b} / N_{0}=$ $5 \mathrm{~dB}$, when supporting $K=3$ users, each employing $N_{k}=2$ receive antennas over a flat-fading MIMO channel. Our future research will consider the employment of sphere-packing-aided [15] multilevel modulation schemes that are protected by the iteratively detected bit-interleaved coded modulation and the turbo trellis-coded modulation [6].

\section{REFERENCES}

[1] S. X. Ng and L. Hanzo, "On the MIMO channel capacity of multidimensional signal sets," IEEE Trans. Veh. Technol., vol. 55, no. 2, pp. 528-536, Mar. 2006.

[2] D. Tse, P. Viswanath, and L. Zheng, "Diversity-multiplexing tradeoff in multiple-access channels," IEEE Trans. Inf. Theory, vol. 50, no. 9, pp. $1859-1874$, Sep. 2004.

[3] L. Hanzo, M. Münster, B.-J. Choi, and T. Keller, OFDM and MC-CDMA for Broadband Multi-User Communications, WLANs and Broadcasting. Piscataway, NJ: IEEE Press, 2003.

[4] P. Vandenameele, L. van der Perre, and M. Engels, Space Division Multiple Access for Wireless Local Area Networks. Norwell, MA: Kluwer, 2001.

[5] J. Blogh and L. Hanzo, $3 G$ Systems and Intelligent Networking. Piscataway, NJ: IEEE Press, 2002. [Online]. Available: http://wwwmobile.ecs.soton.ac.uk

[6] L. Hanzo, T. Liew, and B. Yeap, Turbo Coding, Turbo Equalisation and Space-Time Coding. Piscataway, NJ: IEEE Press, 2002. [Online]. Available: http://www-mobile.ecs.soton.ac.uk

[7] R. Irmer, "Multiuser transmission in code division multiple access mobile communications systems," Ph.D. dissertation, Technische Universitat Dresden, Dresden, Germany, 2005.

[8] L.-U. Choi and R. Murch, "A transmit preprocessing technique for multiuser MIMO systems using a decomposition approach," IEEE Trans. Wireless Commun., vol. 3, no. 1, pp. 20-24, Jan. 2004.

[9] D. Divsalar, S. Dolinar, and F. Pollara, "Serial turbo trellis coded modulation with rate-1 inner code," in Proc. IEEE GLOBECOM, 2000, vol. 2, pp. 777-782.

[10] Q. Spencer, A. Swindlehurst, and M. Haardt, "Zero-forcing methods for downlink spatial multiplexing in multiuser MIMO channels," IEEE Trans. Signal Process., vol. 52, no. 2, pp. 461-471, Feb. 2004.

[11] L. Hanzo and T. Keller, OFDM and MC-CDMA: A Primer. Piscataway, NJ: IEEE Press, 2006.

[12] D. Pham, K. Pattipati, P. Willet, and J. Luo, "An improved complex sphere decoder for v-blast systems," IEEE Signal Process. Lett., vol. 11, no. 19, pp. 748-751, Sep. 2004.

[13] J. E. Gentle, Numerical Linear Algebra for Applications in Statistics. Berlin, Germany: Springer-Verlag, 1998.

[14] S. Ten Brink, "Designing iterative decoding schemes with the extrinsic information transfer chart," $A E U$, Int. J. Electron. Commun., vol. 54, no. 6, pp. 389-398, Nov. 2000.

[15] O. R. Alamri, B. L. Yeap, and L. Hanzo, "A turbo detection and sphere-packing-modulated-aided space-time coding scheme," IEEE Trans. Veh. Technol., vol. 56, no. 2, pp. 575-582, Mar. 2007. 